\title{
He's variational iteration method for nonlinear jerk equations: Simple but effective
}

\author{
Behrouz Raftari \\ Department of Mathematics, Kermanshah Branch, Islamic Azad University, Kermanshah, Iran \\ E-mail: behrouz.raftari@gmail.com
}

Received 9 March 2012

Revised 24 September 2012

Accepted 3 October 2012

\begin{abstract}
The variational iteration method (VIM) for determining periodic solutions of non-linear jerk equations involving third-order time-derivative is presented. This method is based on the use of Lagrange multipliers for identification of optimal value of a parameter in a functional. We propose first-order approximate VIM solution for this equation and we compare this solution with the solution obtained by harmonic balance method.
\end{abstract}

Keywords: Nonlinear jerk equations, variational iteration method, periodic solutions

\section{Introduction}

Nonlinear phenomena are of fundamental importance in various fields of science and engineering. Most nonlinear phenomena are models of our real-life problems. The world around us can be modeled in terms of nonlinear equations. Nonlinear equations are widely used to describe complex physical phenomena in various fields of sciences, especially in fluid mechanics, solid state physics, plasma physics, plasma wave and chemical physics. The wide applicability of these equations is the main reason why they have attracted so much attention from many mathematicians. However, they are usually very difficult to solve, either numerically or theoretically.

Variational iteration method was first proposed by the Chinese mathematician He [1]. The VIM is shown to effectively, easily and accurately solve a large class of nonlinear problems with components converging rapidly to accurate solutions. For concrete problems, a few approximations can be used for numerical purposes with a high degree of accuracy. The VIM uses only the initial conditions and does not require specific transformations for nonlinear terms as required by some existing techniques.

Nonlinear third-order differential equations (nonlinear jerk equations) can describe some physical problems [6] such as third order mechanical oscillators [7]. In this paper, we consider a Jerk equation of the form

$$
x^{\prime \prime \prime}=J\left(x, x^{\prime}, x^{\prime \prime}\right)
$$

with initial conditions

$$
x(0)=0, \quad x^{\prime}(0)=B, \quad x^{\prime \prime}(0)=0 .
$$

Recently researchers obtained analytical approximations of periodic solutions to Eq. (1) by harmonic balance (HB) method [6,8], homotopy perturbation method (HPM) [9], perturbation method [10], Mickens iteration procedure [11] and homotopy analysis method [12]. The purpose of this paper is to apply variational iteration method which is useful for finding an periodic solution of non-linear jerk equations. 


\section{Variational iteration method}

To illustrate the basic concepts of the variational iteration method, we consider the following differential equation:

$$
L(x)+N(x)=g(t),
$$

where $L$ is a linear operator, $N$ is a nonlinear operator and $g(t)$ is an inhomogeneous term. Then we can construct a correction functional as follows:

$$
x_{n+1}(t)=x_{n}(t)+\int_{0}^{t} \lambda\left\{L x_{n}(\tau)+N \tilde{x}_{n}(\tau)-g(\tau)\right\} d \tau,
$$

where $\lambda$ is a general Lagrange multiplier, which can be identified optimally via variational theory. The second term on the right hand side is called the correction and is considered as a restricted variation, i.e, $\delta \tilde{x}_{n}=0$.

By determining $\lambda$, the approximations $x_{n}(t), n \geqslant 0$ obtain immediately. Consequently, the exact solution may be obtained by

$$
x(t)=\lim _{n \rightarrow \infty} x_{n}(t) .
$$

We consider the Eq. (1) subject to the initial condition Eq. (2) and we rewrite this equation as following form:

$$
x^{\prime \prime \prime}+\Omega^{2} x^{\prime}-J\left(x, x^{\prime}, x^{\prime \prime}\right)-\Omega^{2} x^{\prime}=0,
$$

According to the variational iteration method, we can construct the following correction functional:

$$
x_{n+1}(t)=x_{n}(t)+\int_{0}^{t} \lambda(\tau)\left\{x_{n}^{\prime \prime \prime}(\tau)+\Omega^{2} x_{n}^{\prime}(\tau)-J\left(\tilde{x}_{n}(\tau), \tilde{x}_{n}^{\prime}(\tau), \tilde{x}_{n}^{\prime \prime}(\tau)\right)-\Omega^{2} \tilde{x}_{n}^{\prime}(\tau)\right\} d \tau,
$$

Making the above correction functional stationary, and noticing that $\delta \tilde{x}_{n}=0$, we have

$$
\begin{aligned}
\delta x_{n+1}(t)= & \delta x_{n}(t)+\delta \int_{0}^{t} \lambda(\tau)\left\{x_{n}^{\prime \prime \prime}(\tau)+\Omega^{2} x_{n}^{\prime}(\tau)-J\left(\tilde{x}_{n}(\tau), \tilde{x}_{n}^{\prime}(\tau), \tilde{x}_{n}^{\prime \prime}(\tau)\right)-\Omega^{2} \tilde{x}_{n}^{\prime}(\tau)\right\} d \tau, \\
= & \delta x_{n}(t)+\left.\lambda(\tau) \delta x_{n}^{\prime \prime}(\tau)\right|_{\tau=t}-\left.\lambda^{\prime}(\tau) \delta x_{n}^{\prime}(\tau)\right|_{\tau=t}+\left.\left(\lambda^{\prime \prime}(\tau)+\Omega^{2} \lambda(\tau)\right) \delta x_{n}(\tau)\right|_{\tau=t} \\
& -\delta \int_{0}^{t}\left(\lambda^{\prime \prime \prime}(\tau)+\Omega^{2} \lambda^{\prime}(\tau)\right) x_{n}(\tau) d \tau,
\end{aligned}
$$

this yields the following stationary conditions

$$
\begin{aligned}
& \delta x_{n}(t): \lambda^{\prime \prime}(t)+\Omega^{2} \lambda(t)+1=0, \\
& \delta x_{n}^{\prime \prime}(t): \lambda(t)=0 \\
& \delta x_{n}^{\prime}(t): \lambda^{\prime}(t)=0 \\
& \delta x_{n}(\tau): \lambda^{\prime \prime \prime}(\tau)+\Omega^{2} \lambda^{\prime}(\tau)=0,
\end{aligned}
$$

Therefore, the Lagrange multiplier can be readily identified as

$$
\lambda(\tau)=\frac{1}{\Omega^{2}}(\cos \Omega(\tau-t)-1) .
$$

Substituting this value of the Lagrangian multiplier into functional Eq. (7) gives

$$
\begin{aligned}
& x_{n+1}(t)=x_{n}(t)+\int_{0}^{t} \frac{1}{\Omega^{2}}(\cos \Omega(\tau-t)-1) \\
& \left\{x_{n}^{\prime \prime \prime}(\tau)+\Omega^{2} x_{n}^{\prime}(\tau)-J\left(x_{n}(\tau), x_{n}^{\prime}(\tau), x_{n}^{\prime \prime}(\tau)\right)-\Omega^{2} x_{n}^{\prime}(\tau)\right\} d \tau .
\end{aligned}
$$

Regarding to the new structure of VIM proposed by He [2-5], for solving the jerk equation we consider the following formula:

$$
x_{n+1}(t)=x_{0}(t)+\int_{0}^{t} \frac{1}{\Omega^{2}}(\cos \Omega(\tau-t)-1)\left\{-J\left(x_{n}(\tau), x_{n}^{\prime}(\tau), x_{n}^{\prime \prime}(\tau)\right)-\Omega^{2} x_{n}^{\prime}(\tau)\right\} d \tau .
$$


Now from the initial conditions (2), we begin with initial approximation

$$
x_{0}=\frac{B}{\Omega} \sin (\Omega t),
$$

where $\Omega$ is the angular frequency. By the above iteration formula, we can obtain $x_{n}(t)$ for $n \geqslant 1$. In the present work, we consider first-order solution $\left(x_{1}(t)\right)$ for the studied problem and we only consider the following cubic nonlinear functions desired by Gottlieb: (I) $x x^{\prime} x^{\prime \prime}$; (II) $x^{\prime} x^{\prime \prime 2}$; (III) $x^{2} x^{\prime}$ and (IV) $x^{\prime 3}$.

\section{General nonlinear Jerk function}

The most general Jerk function, which is invariant under time-reversal and space-reversal and only cubic nonlinear as specified above, may be written as

$$
x^{\prime \prime \prime}+\alpha x x^{\prime} x^{\prime \prime}+\beta x^{\prime} x^{\prime \prime 2}+\delta x^{2} x^{\prime}+\varepsilon x^{\prime 3}+\gamma x^{\prime}=0,
$$

where the parameters $\alpha, \beta, \delta, \varepsilon$ and $\gamma$ are constants.

\subsection{Jerk function containing displacement times velocity times acceleration, and velocity}

For function (I), and a linear term in the velocity is incorporated. The resulting standardized Jerk equation, after rescaling of both $x$ and $t$, is taken to be

$$
x^{\prime \prime \prime}=x x^{\prime} x^{\prime \prime}-x^{\prime},
$$

with initial conditions

$$
x(0)=0, \quad x^{\prime}(0)=B, \quad x^{\prime \prime}(0)=0,
$$

Assume that the angular frequency is $\Omega$, with the period $T$ given by

$$
T=\frac{2 \pi}{\Omega}
$$

Now, we illustrate the solution of this problem by variational iteration method. From the iteration formula (12), we have

$$
x_{1}(t)=x_{0}(t)+\int_{0}^{t} \frac{1}{\Omega^{2}}(\cos \Omega(\tau-t)-1)\left\{-x_{0}(\tau) x_{0}^{\prime}(\tau) x_{0}^{\prime \prime}(\tau)+x_{0}^{\prime}(\tau)-\Omega^{2} x_{0}^{\prime}(\tau)\right\} d \tau,
$$

then

$$
x_{1}(t)=\frac{B}{\Omega} \sin (\Omega t)+\frac{B}{96 \Omega^{3}} \times\left(\left(-9 B^{2}-48+48 \Omega^{2}\right) \sin (\Omega t)+\left(12 \Omega B^{2}+48 \Omega-48 \Omega^{3}\right) \operatorname{tcos}(\Omega t)-B^{2} \sin (3 \Omega t)\right)
$$

Now He recommends that the adjustable parameter must be so chosen that the solution may be free of the secular term. This can be ensured if we choose

$$
12 \Omega B^{2}+48 \Omega-48 \Omega^{3}=0,
$$

then, we find

$$
\Omega=\frac{1}{2} \sqrt{B^{2}+4}
$$

It is in agreement with Gottlieb's result[6].

The periodic solution of Eq. (15) can be obtained as

$$
x(t)=\frac{B}{\Omega} \sin (\Omega t)+\frac{B}{96 \Omega^{3}}\left(\left(-9 B^{2}-48+48 \Omega^{2}\right) \sin (\Omega t)-B^{2} \sin (3 \Omega t)\right) .
$$




\subsection{Jerk function containing velocity-cubed and velocity times displacement-squared}

For functions (III) and (IV), after rescaling of both $x$ and $t$, the corresponding standardized Jerk equation would take the form

$$
x^{\prime \prime \prime}=-x^{\prime}\left(x^{2}+x^{2}\right),
$$

with the initial conditions

$$
x(0)=0, \quad x^{\prime}(0)=B, \quad x^{\prime \prime}(0)=0 .
$$

Assume that the angular frequency is $\Omega$, with the period $T$ given by

$$
T=\frac{2 \pi}{\Omega}
$$

By the iteration formulae (12), we have

$$
x_{1}(t)=x_{0}(t)+\int_{0}^{t} \frac{1}{\Omega^{2}}(\cos \Omega(\tau-t)-1)\left\{x_{0}^{\prime}(\tau)\left(x_{0}^{\prime}(\tau)^{2}+x_{0}(\tau)^{2}\right)-\Omega^{2} x_{0}^{\prime}(\tau)\right\} d \tau,
$$

then

$$
\begin{aligned}
x_{1}(t)= & \frac{B}{\Omega} \sin (\Omega t)+\frac{B}{96 \Omega^{5}}\left(\left(-9 B^{2}+48 \Omega^{4}-39 B^{2} \Omega^{2}\right) \sin (\Omega t)\right. \\
& \left.+\left(12 \Omega B^{2}-48 \Omega^{5} t+36 \Omega^{3} B^{2}\right) \operatorname{tcos}(\Omega t)+\left(B^{2} w^{2}-B^{2}\right) \sin (3 \Omega t)\right),
\end{aligned}
$$

where the angular frequency $\Omega$ is identified with the physical understanding that no secular terms should be appeared in $x_{1}(t)$, which leads to

$$
12 \Omega B^{2}-48 \Omega^{5} t+36 \Omega^{3} B^{2}=0,
$$

then, we find

$$
\Omega=\frac{1}{4} \sqrt{6 B^{2}+2 B \sqrt{9 B^{2}+16}}
$$

This value equal with value obtained by harmonic balance method [6].

The periodic solution of Eq. (23) can be obtained as

$$
x_{1}(t)=\frac{B}{\Omega} \sin (\Omega t)+\frac{B}{96 \Omega^{5}} \times\left(\left(-9 B^{2}+48 \Omega^{4}-39 B^{2} \Omega^{2}\right) \sin (\Omega t)+\left(B^{2} w^{2}-B^{2}\right) \sin (3 \Omega t)\right),
$$

\subsection{Jerk function containing velocity times acceleration-squared, and velocity}

For function (II), a linear term in the velocity is incorporated. The resulting standardized Jerk equation, after rescaling of both $x$ and $t$, is taken to be

$$
x^{\prime \prime \prime}=-x^{\prime} x^{\prime \prime 2}-\gamma x^{\prime},
$$

with the initial conditions

$$
x(0)=0, \quad x^{\prime}(0)=B, \quad x^{\prime \prime}(0)=0 .
$$

Assume that the angular frequency is $\Omega$, with the period $T$ given by

$$
T=\frac{2 \pi}{\Omega}
$$

In this case, we have following iteration formula

$$
x_{1}(t)=x_{0}(t)+\int_{0}^{t} \frac{1}{\Omega^{2}}(\cos \Omega(\tau-t)-1)\left\{x_{0}^{\prime}(\tau) x_{0}^{\prime \prime}(\tau)^{2}+\gamma x_{0}(\tau)^{\prime}-\Omega^{2} x_{0}^{\prime}(\tau)\right\} d \tau .
$$

By the iteration formulae (34), we have 


$$
\begin{aligned}
x_{1}(t)= & \frac{B}{\Omega} \sin (\Omega t)+\frac{B}{96 \Omega^{3}}\left(\left(-9 B^{2} \Omega^{2}-48 \gamma+48 \Omega^{2}\right) \sin (\Omega t)\right. \\
& \left.+\left(12 \Omega^{3} B^{2}+48 \gamma \Omega-48 \Omega^{3}\right) \operatorname{tcos}(\Omega t)-B^{2} \Omega^{2} \sin (3 \Omega t)\right)
\end{aligned}
$$

where the angular frequency is identified with the understanding that no secular terms occur, which leads to

$$
12 \Omega^{3} B^{2}+48 \gamma \Omega-48 \Omega^{3}=0,
$$

then, we find

$$
\Omega=2 \sqrt{\frac{\gamma}{4-B^{2}}}
$$

It is in agreement with result obtained by harmonic balance method [6].

The periodic solution of Eq. (31) can be obtained as

$$
x_{1}(t)=\frac{B}{\Omega} \sin (\Omega t)+\frac{B}{96 \Omega^{3}}\left(\left(-9 B^{2} \Omega^{2}-48 \gamma+48 \Omega^{2}\right) \sin (\Omega t)-B^{2} \Omega^{2} \sin (3 \Omega t)\right) .
$$

\subsection{Jerk function containing velocity-cubed, velocity times displacement-squared, and velocity}

For functions (III) and (IV), a linear term in the velocity is incorporated. The resulting standardized Jerk equation, after rescaling of both $x$ and $t$, is taken to be

$$
x^{\prime \prime \prime}=-x^{\prime}\left(x^{2}+x^{2}\right)-\gamma x^{\prime},
$$

with the initial conditions

$$
x(0)=0, \quad x^{\prime}(0)=B, \quad x^{\prime \prime}(0)=0 .
$$

Assume that the angular frequency is $\Omega$, with the period $T$ given by

$$
T=\frac{2 \pi}{\Omega}
$$

By the iteration formulae (12), we have

$$
x_{1}(t)=x_{0}(t)+\int_{0}^{t} \frac{1}{\Omega^{2}}(\cos \Omega(\tau-t)-1)\left\{x_{0}^{\prime}(\tau)\left(x_{0}^{\prime}(\tau)^{2}+x_{0}(\tau)^{2}\right)+\gamma x^{\prime}-\Omega^{2} x_{0}^{\prime}(\tau)\right\} d \tau,
$$

then

$$
\begin{aligned}
x_{1}(t)= & \frac{B}{\Omega} \sin (\Omega t)+\frac{B}{96 \Omega^{5}}\left(\left(-39 B^{2} \Omega^{2}-9 B^{2}-48 \gamma \Omega^{2}+48 \Omega^{4}\right) \sin (\Omega t)\right. \\
& \left.+\left(36 \Omega^{3} B^{2}+12 \Omega B^{2}+48 \gamma \Omega^{3}-48 \Omega^{5}\right) \operatorname{tcos}(\Omega t)+B^{2}\left(\Omega^{2}-1\right) \sin (3 \Omega t)\right)
\end{aligned}
$$

where the angular frequency is identified with the understanding that no secular terms occur, which leads to

$$
36 \Omega^{3} B^{2}+12 \Omega B^{2}+48 \gamma \Omega^{3}-48 \Omega^{5}=0,
$$

then, we find

$$
\Omega=\frac{1}{4} \sqrt{6 B^{2}+8 \gamma+2 \sqrt{9 B^{4}+24 B^{2} \gamma+16 \gamma^{2}+16 B^{2}}}
$$

this value for $\Omega$ in this case is agreement with the solution obtained by Gottlieb [6].

Then the periodic solution of Eq. (39) can be obtained as

$$
x_{1}(t)=\frac{B}{\Omega} \sin (\Omega t)+\frac{B}{96 \Omega^{5}}\left(\left(-39 B^{2} \Omega^{2}-9 B^{2}-48 \gamma \Omega^{2}+48 \Omega^{4}\right) \sin (\Omega t)+B^{2}\left(\Omega^{2}-1\right) \sin (3 \Omega t)\right)
$$




\section{Discussion}

In this paper, the Jerk equations involving the third temporal derivative of displacement are analyzed by special version of variational iteration method. For the same cases as discussed by Gottlieb [6], the results for periods were compared with the results obtained by the harmonic balance method. It is found that the first-order approximation is identical to the result obtained by the harmonic balance method. In this study, we found a new Lagrange multiplier for VIM formulation which is suitable for the nonlinear ODE with the following form

$$
u^{\prime \prime \prime}=F\left(u, u^{\prime}, u^{\prime \prime}, \ldots, u^{(n)}\right)
$$

\section{References}

[1] J.H. He, Variational iteration method - a kind of non-linear analytical technique: some examples, International Journal of Non-linear Mechanics 34 (1999), 699-708.

[2] J.H. He, Variational iteration method - Some recent results and new interpretations, Journal of Computational and Applied Mathematics 207(1) (2007), 3-17.

[3] J.H. He and X.H. Wu, Variational iteration method: New development and applications, Computers \& Mathematics with Applications 54 (2007), 881-894.

[4] H.H. He, Some asymptotic methods for strongly nonlinear equations, International Journal of Modern Physics B 20(10) (2006), $1141-1199$.

[5] J.H. He, A short remark on fractional variational iteration method, Physics Letters A 375(38) (2011), 3362-3364.

[6] H.P.W. Gottlieb, Harmonic balance approach to periodic solutions of non-linear Jerk equations, Journal of Sound and Vibration 271(35) (2004), 671-683.

[7] Y.A. Mitropolskii and N.V. Dao, Applied Asymptotic Methods in Nonlinear Oscillations, Kluwer Academic Publishers, Dordrecht, 1997.

[8] H.P.W. Gottlieb, Harmonic balance approach to limit cycles for nonlinear Jerk equations, Journal of Sound and Vibration 297(12) (2006), $243-250$.

[9] X. Ma, L. Wei and Z. Guo, He's homotopy perturbation method to periodic solutions of nonlinear Jerk equations, Journal of Sound and Vibration 314 (2008), 217-227.

[10] H. Hu, Perturbation method for periodic solutions of nonlinear jerk equations, Physics Letters A 372 (2008), 4205-4209.

[11] H. Hu, M.Y. Zheng and Y.J. Guo, Iteration calculations of periodic solutions to nonlinear jerk equations, Acta Mech 209 (2010), $269-274$.

[12] S.D. Feng and L.-Q. Chen, Homotopy Analysis Approach to Periodic Solutions of a Nonlinear Jerk Equation, Chin Phys Lett 26(12) (2009), 124501. 

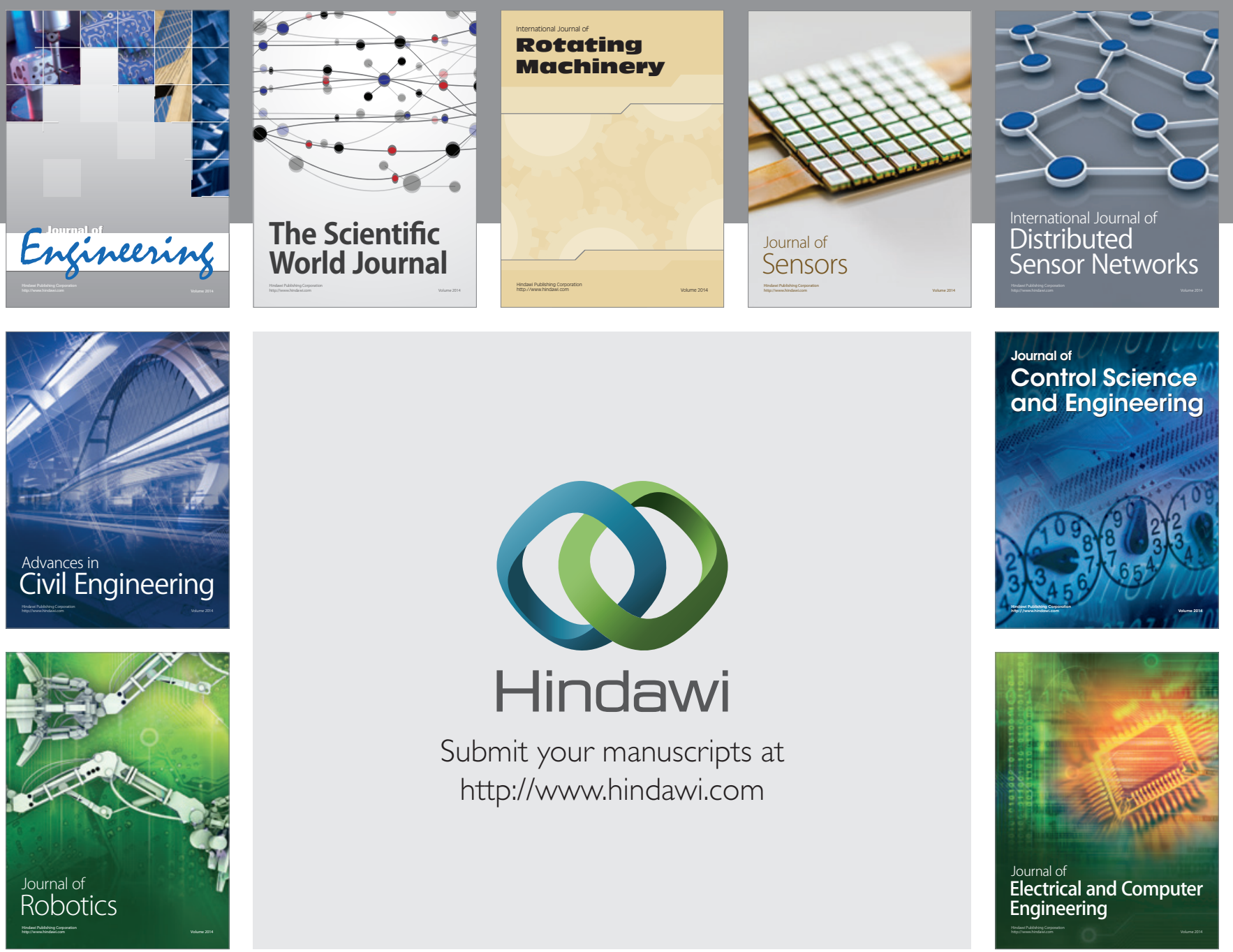

Submit your manuscripts at

http://www.hindawi.com
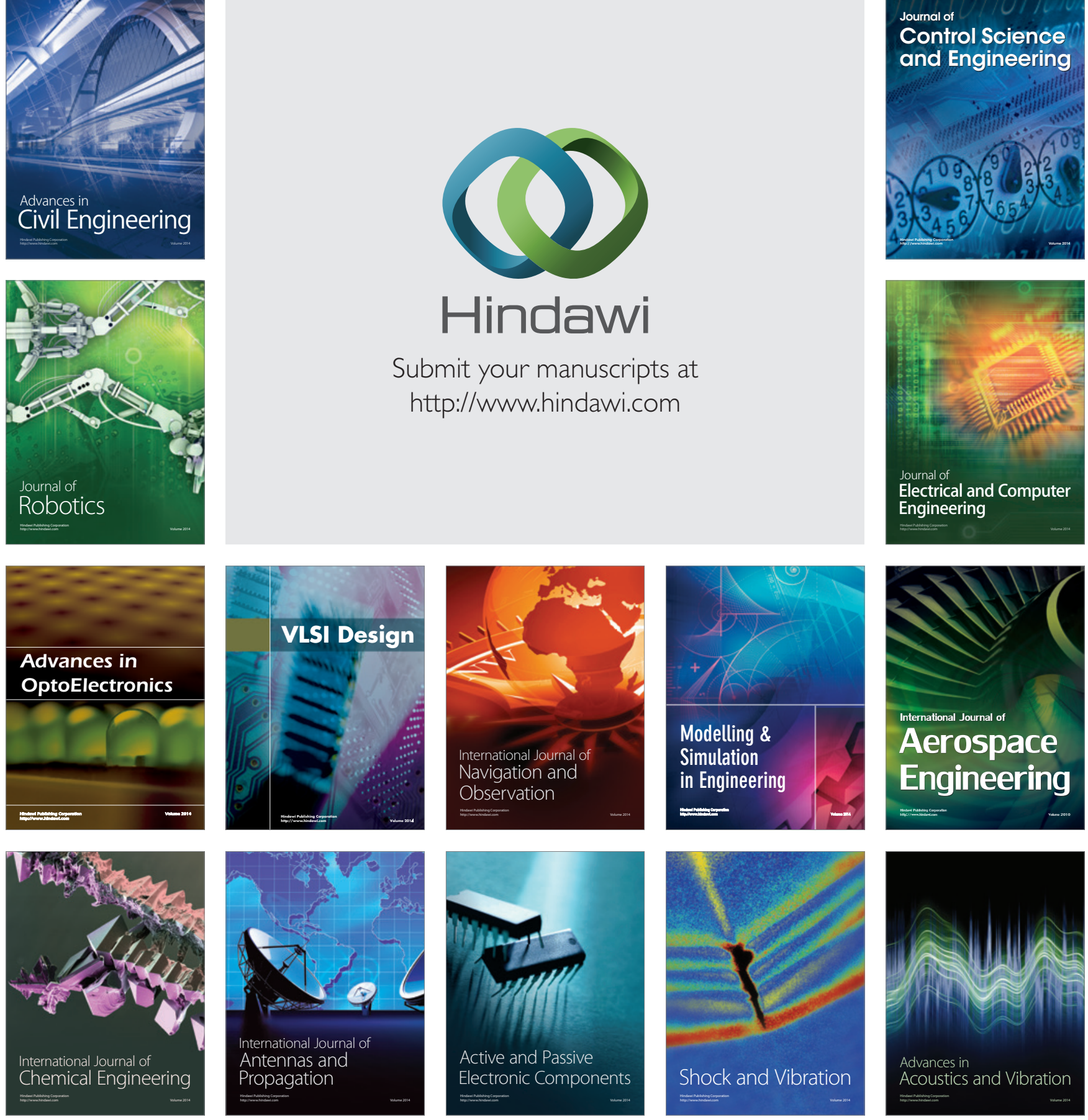